\title{
Hepatotoxicity induced by antifungal drugs itraconazole and fluconazole in rats: a comparative in vivo study
}

\begin{abstract}
Itraconazole and fluconazole are oral antifungal drugs, which have a wide spectrum antifungal activity and better efficacy than the older drugs. However, both drugs have been associated with hepatotoxicity in susceptible patients. The mechanism of antifungal druginduced hepatotoxicity is largely unknown. Therefore, the aim of this present study was to investigate and compare the hepatotoxicity induced by these drugs in vivo. Rats were treated intraperitoneally with itraconazole or fluconazole either single $(0,10,100$ and $200 \mathrm{mg} / \mathrm{kg}$ ) or subchronic $(0,10,50$ and $100 \mathrm{mg} / \mathrm{kg}$ per day for 14 days) doses. Plasma and liver samples were taken at the end of the study. A statistically significant and dose dependent increase of plasma alanine aminotransferase (ALT) and alkaline phosphatase (ALP) activities were detected in the subchronic itraconazole-treated group. In addition, dose-dependent hepatocellular necrosis, degeneration of periacinar and mizonal hepatocytes, bile duct hyperplasia and biliary cirrhosis and giant cell granulome were observed histologically in the same group. Interestingly, fluconazole treated rats had no significant increase in transaminases for both single and subchronic groups. In the subchronic fluconazole treated rats, only mild degenerative changes of centrilobular hepatocytes were observed. These results demonstrated that itraconazole was a more potent hepatotoxicant than fluconazole in vivo in rats.
\end{abstract}

Keyword: Fluconazole; Hepatotoxicity; Itraconazole; Rats 